\title{
SINTOMAS DEPRESSIVOS EM ESCOLARES: ESTUDO EXPLORATÓRIO EM UM SERVIÇO ESCOLA
}

Bruna Rafaele Milhorini Greinert ${ }^{1}$, Amanda Cristina Pereira Ramos de Souza ${ }^{2}$, Natane Bianca da Silva ${ }^{2}$, Regina Karisa de Freitas ${ }^{2}$, Rute Grossi Milani ${ }^{3}$

1 Mestranda do Programa de Mestrado em Promoção da Saúde do Centro Universitário Cesumar - UniCesumar - Maringá - Pr, Brasil.

(brunamilhorini@hotmail.com).

2 Acadêmicas do curso de Psicologia do Centro Universitário Cesumar UniCesumar - Maringá - Pr, Brasil.

3 Profa Dra do Programa de Mestrado em Promoção da Saúde e do Programa de Mestrado em Tecnologias Limpas do Centro Universitário Cesumar - UniCesumar Maringá - Pr, Brasil; Pesquisadora do Instituto Cesumar de Ciência, Tecnologia e Inovação (ICETI)

Recebido em: 03/10/2016 - Aprovado em: 21/11/2016 - Publicado em: 05/12/2016 DOI: 10.18677/EnciBio_2016B_139

\section{RESUMO}

O presente estudo visou caracterizar os casos de crianças atendidas por uma clínica escola de psicologia com relação aos sintomas depressivos e fatores adversos relacionados. Efetuou-se um levantamento nos prontuários de crianças com idade de seis a 12 anos, no período de 2011 a 2012. Em relação aos sintomas, observouse uma variada gama de manifestações com características depressivas entre as crianças atendidas. Os sintomas depressivos mais presentes foram a falta de interesse em atividades escolares, dificuldade de reter informações, sentimento de tristeza, choro sem motivo, isolamento social e timidez. O principal fator adverso presente no histórico de vida das crianças foi a ausência do pai ou da mãe. Os achados da pesquisa sinalizam a necessidade de que a escola e os serviços de saúde estejam preparados para reconhecerem os sintomas da depressão infantil, que tendem a se manifestar no ambiente escolar, buscando implantar ações de promoção da saúde e o tratamento adequado à criança em desenvolvimento. Destaca-se, também, a importância do suporte familiar como um fator protetivo à depressão infantil.

PALAVRAS-CHAVE: bem-estar da criança, depressão infantil, fator de risco.

\section{DEPRESSIVE SYMPTOMS IN SCHOLARSHIPS: EXPLORATORY STUDY IN A SCHOOL'S SERVICE.}

\begin{abstract}
This study aimed to characterize cases of children assisted by a clinical psychological school with regard to depressive symptoms and related adverse factors. We conducted a survey on the medical records of children aged 6 to 12 years, ranging from 2011 to 2012 . With relation to the symptoms, there was a wide range of events with depressive features among the children served. The most present depressive symptoms were lack of interest in school activities, difficulty in information retaining, feelings of sadness, crying for no reason, social isolation and
\end{abstract}


shyness. The main adverse factor present in the children's life history was the absence of the father or mother. The research findings indicate the need for school and health services are prepared to recognize the symptoms of childhood depression, which tend to manifest themselves in the school environment, seeking to implement actions to promote health and proper treatment to the child in development. It's also important to emphasize the familiar support as a protective factor for childhood depression.

KEYWORDS: child welfare, child depression, risk factor.

\section{INTRODUÇÃO}

A depressão é caracterizada como um transtorno de humor e pode acometer indivíduos em qualquer fase da vida. Segundo SILVA \& LACERDA (2014) é significativo o aumento de transtornos mentais que afetam a sociedade atual, em especial, a depressão. Embora esta patologia ao longo dos anos tenha sido comumente associada à fase adulta, são inúmeros os estudos que revelam a incidência da depressão na infância e buscam estudá-la nesta fase da vida (CARVALHO \& RAMIRES, 2013; MIRANDA et al., 2013; SILVA \& LACERDA, 2014;).

As primeiras tentativas de estudar a depressão infantil registradas na literatura surgiram no início do século XIX. No entanto, quanto à conceituação da depressão infantil, deu-se, inicialmente, a partir de um enfoque psicanalítico, objetivando à compreensão psicodinâmica de pessoas deprimidas (MIRANDA et al., 2013). Somente a partir da década de 70 a depressão infantil veio a ser reconhecida, pois até essa época estudiosos pensavam não existir este transtorno em crianças e adolescentes (COLAVITE et al., 2013).

Segundo o APA-DSM-5 (2014), a depressão infantil apresenta-se de forma semelhante à depressão no adulto, pois os mesmos critérios para avaliar a patologia no adulto são utilizados para diagnosticar o transtorno na criança. De acordo com este manual, caracteriza-se como sintomas de depressão: perda de interesse ou prazer, humor deprimido, alterações de apetite e peso, insônia ou hipersonia, fadiga, sentimentos de culpa e inutilidade, dificuldade para se concentrar, falta de energia, pensamentos recorrentes de morte ou tentativas de suicídio. O presente manual também salienta que crianças podem apresentar um humor irritável ou rabugento, em vez de um humor triste ou abatido.

CARVALHO \& RAMIRES (2013) afirmam que é comum em crianças préescolares a depressão ser representada por sintomas físicos, especialmente pela tontura, fadiga, dores de cabeça e abdominais. A criança também pode apresentar crises de ansiedade, que são expressas por meio de fobias, hiperatividade ou agitação psicomotora, irritabilidade, diminuição no apetite, ansiedade de separação e alterações do sono. A enurese e a encoprese também estão frequentemente associadas à depressão infantil, assim como o comportamento agressivo e destrutivo da criança, sua comunicação deficiente, semblante triste e choro constante.

$\mathrm{Na}$ sociedade atual compartilha-se o mito da infância feliz, porém estudos mostram que nem sempre ocorre desta maneira, as crianças têm maior dificuldade em expressar o que estão sentindo na forma verbal (FERNANDES \& MILANI, 2010). Segundo MIRANDA et al. (2013), realizar o diagnóstico da depressão infantil pode ser mais difícil do que diagnosticar a patologia em adultos, uma vez que estes conseguem expressar à família que algo não está bem por meio de suas queixas e ações. Já a criança, por não reconhecer os sintomas como parte de uma doença, a aceita como característica natural do seu modo de ser e existir, pois não 
compreende que há algo errado e que seu sofrimento pode ser aliviado. Portanto, os pais e cuidadores devem estar atentos as mudanças de comportamento da criança para facilitar a identificação precoce dos sintomas depressivos e evitar 0 agravamento do quadro, uma vez que este pode afetar seu convívio social, familiar e escolar. Segundo BENEVIDES et al. (2015), a depressão acarreta um elevado impacto na vida do sujeito, pois compromete inúmeras áreas e contextos da vida da pessoa.

De acordo com CRUVINEL \& BORUCHOVITCH (2003), é preciso considerar que dificilmente um único modelo ou teoria poderá explicar um fenômeno tão delicado e complexo como a depressão, já que este transtorno aborda fatores psicológicos, biológicos, familiares e sociais. Segundo os autores, as diversas teorias existentes sobre a depressão devem contribuir para a compreensão da natureza multicausal da patologia e para a concepção do sujeito em sua totalidade biopsicossocial.

A depressão infantil pode ser desencadeada por fatores biológicos, uma vez que a patologia está associada a alterações químicas no cérebro, e estas são influenciadas por fatores genéticos, sociais e psicológicos. Porém, a depressão pode estar relacionada a fatores externos, como o estresse ou as perdas vivenciadas pela criança. Esses fatores são conhecidos como efeito gatilho (SILVA \& LACERDA, 2014).

A família também possui grande importância no desenvolvimento emocional e social infantil, uma vez que os pais são a primeira referência afetiva e influenciam diretamente a maneira como a criança irá vivenciar possíveis conflitos e eventos estressores que permeiam seu cotidiano. Quando a própria família é incapaz de lidar com situações estressoras, torna-se mais difícil para a criança consegui-lo (RAMOS, 2015). Percebe-se a importância de que a família funcione de forma saudável, capaz de acolher e proteger a criança desde seu nascimento, proporcionando-lhe um ambiente suficientemente bom para seu desenvolvimento e crescimento. Segundo a teoria de Winnicott, todo indivíduo possui um potencial para o desenvolvimento biológico e psicológico, porém se o meio em que está inserido não for suficientemente bom a ponto de não satisfazer suas necessidades, todo este potencial estará comprometido (PERANDRÉA, 2015).

Fica evidente que o contexto familiar é quem fornece a base para a construção da personalidade do sujeito, proporcionando-lhe as primeiras experiências de aprendizagem e dando-lhe condições necessárias para seu crescimento. Para MONBELLI et al. (2011), os conflitos familiares, as infelicidades conjugais $e$ as oscilações emocionais dos pais interferem drasticamente no dinamismo familiar. Segundo os autores, um funcionamento familiar saudável, permeado pelo bom vínculo afetivo, boa comunicação entre os integrantes e coesão familiar configuram-se como fatores de proteção contra o surgimento de distúrbios emocionais. No entanto, apesar da relevância que o ambiente familiar exerce sobre o desenvolvimento da depressão infantil, cabe ressaltar que esta patologia também recebe influência de outros fatores externos.

De acordo com CRUVINEL \& BORUCHOVITCH (2014) problemas de relacionamento com colegas e dificuldade de relacionamento com o professor podem ocasionar a depressão na criança. Além disso, experiências de perdas significativas e luto também são considerados fatores de risco para 0 desenvolvimento do transtorno (MIRANDA et al., 2013; CRUVINEL \& BORUCHOVITCH, 2014). 
Segundo LIMA (2004), é necessário que o manejo psicoterápico seja realizado o mais breve possível, com a avaliação e definição do tipo de tratamento. Devem-se considerar, para a avaliação do transtorno, prejuízos no funcionamento psicossocial do sujeito, a presença de transtornos psíquicos, maus tratos e falhas na educação. Em relação ao tratamento de depressão infantil, há diferença de tratamento para depressão mais leve e severa, pois no caso das leves são realizados encontros regulares com a criança e seus pais, visando ofertar suporte para avaliar o estresse e melhorar o humor.

Em casos mais severos, há um consenso que seja realizado a combinação de psicoterapia e o uso de medicação antidepressiva. A família deve ser conscientizada sobre as consequências da depressão para o desenvolvimento infantil, pois poderá assim, procurar ajuda precoce e evitar maiores comprometimentos (ANDRIOLA \& CAVALCANTE, 1999). Além do manejo realizado com a criança, é importante que seja realizada a intervenção familiar, uma vez que o ambiente proximal da criança pode ocasionar o desenvolvimento da patologia. Esta tentativa é necessária a fim de conscientizar a família de seu papel e capacitá-la para o cuidado. Tal pressuposto vem ao encontro das formulações de Winnicott, pois este argumentava que para as intervenções terapêuticas alcançarem resultados satisfatórios é necessário a família tornar-se capaz de conter as angústias da criança e protegê-la (FIGUEIREDO \& MOTTA, 2014).

Portanto, é de extrema importância a análise do ambiente familiar para que se possa ter uma melhor compreensão dos fatores envolvidos no desenvolvimento da depressão infantil, ressaltando a importância de uma avaliação criteriosa por parte do profissional, para que, desta forma, seja possível realizar um diagnóstico fidedigno e uma intervenção adequada. É necessário conscientizar familiares, educadores e profissionais de saúde sobre a existência da depressão infantil e a necessidade de se identificar os fatores adversos visando o estabelecimento de estratégias de prevenção e tratamento.

A identificação precoce dos sintomas depressivos e o diagnóstico inicial da depressão infantil podem auxiliar profissionais da saúde a intervir junto à criança e, se possível, no contexto familiar, de forma a permitir a diminuição de seu sofrimento mental e, consequentemente, melhorar sua qualidade de vida, contribuindo assim para a saúde da criança. Portanto, o presente estudo visou caracterizar os casos de crianças atendidas por uma clínica escola de psicologia com relação aos sintomas depressivos e fatores adversos relacionados.

\section{MATERIAL E MÉTODOS}

Trata-se de uma pesquisa documental quantitativa desenvolvida mediante a aprovação do Comitê de Ética em Pesquisa, sob o parecer nำ 415.245. O estudo foi realizado em um serviço escola de uma instituição de ensino superior do noroeste do estado do Paraná, consultando-se os prontuários das crianças atendidas, autorizados previamente pelos pais para uso em pesquisa científica. Selecionou-se como período para consulta os anos de 2011 e 2012. Foi realizado um levantamento dos casos de crianças com idade entre seis e 12 anos que apresentavam sintomas depressivos. Esses prontuários oferecem informações a respeito do paciente e têm como objetivo organizar os procedimentos relacionados ao acompanhamento psicológico.

Primeiramente foram selecionados os prontuários com relatos de sintomas depressivos e, em seguida, efetuou-se a caracterização de tais sintomas e a identificação dos fatores ambientais adversos presentes no histórico de vida dessas 
crianças, esta etapa foi efetuada com base em MARCELLI (1998). Dentre as limitações do presente estudo, destaca-se que no processo de coleta de dados alguns prontuários apresentavam informações incompletas, letras ilegíveis e irregulares, e a maioria não estava autorizado para pesquisa, fator este que restringiu o número de prontuários para análise. É importante salientar que foram analisados os casos que apresentavam sintomas sugestivos de depressão, e não a presença do transtorno em si, já que o diagnóstico nosológico da depressão requer uma investigação clínica profunda e minuciosa.

A análise dos dados foi realizada de forma quantitativa, mediante o uso de estatística descritiva.

\section{RESULTADOS E DISCUSSÕES}

O levantamento dos prontuários do Serviço Escola de Psicologia, referentes ao período de 2011 a 2012, possibilitou identificar 17 prontuários com queixas sugestivas de sintomas depressivos. A idade dos pacientes incluídos na pesquisa variou entre seis e 12 anos, sendo $52 \%$ do sexo feminino e $47 \%$ do sexo masculino.

Em relação aos sintomas, observou-se, pela análise dos prontuários, uma variada gama de manifestações com características depressivas entre as crianças atendidas, porém não houve casos que apresentaram um diagnóstico preciso de depressão infantil. Tal fato justifica-se uma vez que a complexidade da patologia e os diferentes sintomas manifestos tornam mais complexo e delicado realizar o diagnóstico nosológico da depressão. Segundo MIRANDA et al. (2013), é necessário considerar que as manifestações do quadro clínico diferem de pessoa para pessoa, não se apresentando igualmente em todos os sujeitos. ÁLVARES \& LOBATO (2013) salientam que o diagnóstico de depressão requer uma investigação clínica minuciosa e profunda. Por esta razão, nos resultados do presente estudo serão enfocados os sintomas sugestivos de depressão e não a presença do transtorno em si, entendendo-se que esses sintomas também podem estar atrelados a outros quadros psicopatológicos.

Os sintomas depressivos identificados na análise dos 17 prontuários estão demonstrados na tabela 1. Algumas crianças apresentaram mais de um sintoma depressivo.

TABELA 1. Sintomas depressivos em escolares, segundo frequência e porcentagem.

\begin{tabular}{llr}
\hline Sintomas & F & $\%$ \\
\hline Agressividade & 7 & 12,5 \\
\hline Ansiedade & 3 & 5,3 \\
Diminuição do apetite & 2 & 3,5 \\
\hline $\begin{array}{l}\text { Falta de interesse em atividades escolares e } \\
\text { dificuldade de reter informações }\end{array}$ & 9 & 15,9 \\
Hipersonia & 2 & 3,5 \\
Insônia & 3 & 5,3 \\
Irritabilidade e embravecimento & 8 & 14,2 \\
Isolamento social e timidez & 8 & 14,2 \\
Medo de ficar doente e de morrer & 1 & 1,7 \\
Preguiça & 1 & 1,7 \\
Queixas somáticas & 4 & 7,1 \\
Sentimento de tristeza e choro sem motivo & 8 & 14,2 \\
\hline
\end{tabular}

Fonte: Dados de prontuários de um serviço escola de psicologia, no período de 2011 a 2012. 
Os principais sintomas depressivos identificados, de acordo com a tabela 1, foram a falta de interesse em atividades escolares e dificuldade de reter informações com $15,9 \%$, seguido por irritabilidade e embravecimento, sentimento de tristeza e choro sem motivo, isolamento social e timidez, todos com 14,2\%. As queixas somáticas representaram $7,1 \%$ e os sintomas de ansiedade e insônia, ambos, tiveram 5,30\%. Hipersonia, diminuição do apetite, preguiça e medo de ficar doente obtiveram frequência inferior a $4 \%$.

Percebe-se como principal sintoma sugestivo de depressão neste estudo a falta de interesse em atividades escolares e a dificuldade da criança em reter informações. Segundo FERNANDES \& MILANI (2010), a depressão infantil está associada à crença de autoeficácia, uma vez que a criança depressiva possui dificuldade para acreditar em sua própria capacidade, isso consequentemente acarretará prejuízos no rendimento escolar. Segundo as autoras, a depressão afeta áreas do pensamento, das emoções e do comportamento da criança. Uma vez que esta patologia interfere negativamente em diversas áreas da vida da criança, esta tende a não acreditar mais em si mesma e em sua capacidade para transpor obstáculos, perdendo assim a expectativa de obter resultados assertivos independente do investimento que lhe é proposto. Mediante tais condições, torna-se extremamente custoso para a criança prosseguir e avançar em seu desempenho escolar, pois não reconhece em si recursos internos para lidar com os desafios desta fase. Além disso, toda e qualquer criança ao se deparar com as novas exigências provenientes do ambiente acadêmico sente-se pressionada e pode apresentar dificuldades para suprir a demanda do meio. No caso da criança com sintomas depressivos, seu ego encontra-se ainda mais fragilizado, sendo uma tarefa difícil lidar com suas angústias e conflitos.

Notou-se neste estudo que as queixas somáticas foram caracterizadas como sintomas depressivos. Tal fato pode ser explicado pelo mecanismo de transferência, em que a criança manifesta por meio de dores físicas, seja no estômago, dores de cabeça, diarreia e dores no corpo um sintoma emocional que não foi bem elaborado. RAMOS (2015) salienta que as queixas somáticas estão intrinsicamente ligadas com a saúde e aprendizagem da criança, pois quando chega à escola com cefaleia dificilmente conseguirá manter-se concentrada e consequentemente terá seu desempenho escolar afetado. Tal fato pode estar associado à falta de interesse em atividades escolares e à dificuldade de reter informações apresentados pelas crianças neste estudo.

Segundo CARVALHO \& RAMIRES (2013), a irritabilidade também é um sintoma depressivo que as crianças podem apresentar. Foi possível averiguar na presente pesquisa que a irritabilidade e o embravecimento foram caracterizados como sintomas depressivos. Possivelmente tais comportamentos sejam apresentados como um mecanismo de defesa utilizado pela criança por não conseguir lidar com a angústia que permeia seu mundo interno. Por não ser capaz de assimilar e decodificar as emoções, explosões de fúria e irritabilidade são meios utilizados para expressar sua dor psíquica.

Segundo SILVA \& LACERDA (2014), crianças em idade escolar podem apresentar como sintomas depressivos o sentimento de tristeza, choro constante e sem motivo aparente. Os achados deste estudo corroboram tal afirmativa, uma vez que os prontuários analisados apresentaram um alto índice de crianças com sentimento de tristeza e choro sem motivo.

O comportamento agressivo também foi um dos sintomas apresentados pelas crianças. MILANI (2014) adverte que a agressividade pode ser utilizada como 
uma forma de reduzir a angústia causada por um evento estressante, porém tais atitudes podem desencadear consequências negativas à criança, como problemas de relacionamento. Desta forma, pode-se inferir que nos casos de crianças depressivas o uso da força é utilizado na tentativa de controlar estes eventos estressores, a fim de se defenderem de possíveis ameaças a seu ego, que já está fragilizado.

Foi possível verificar que os prontuários analisados revelaram o isolamento social e a timidez também como sintomas depressivos. Segundo SANTOS et al. (2015), crianças com baixo envolvimento social apresentam dificuldade adaptativa frente a novas situações e geralmente reprimem seus impulsos e emoções. Percebe-se que há uma relação entre os sintomas de isolamento social e timidez caracterizados como sugestivos de depressão neste estudo com a pouca flexibilidade da criança para adaptar-se a situações novas. A timidez está relacionada com a repressão que a criança tem de suas emoções e impulsos, na tentativa de contê-los. E o isolamento social, por sua vez, está associado à dificuldade que a criança depressiva possui de enfrentar novos desafios e se relacionar com colegas. Seu isolamento funciona como uma medida protetiva do ego que está fragilizado e não possui condições para interagir com o novo e desconhecido.

Com relação aos fatores adversos que podem levar à depressão infantil, no presente estudo foi considerado que a patologia é um fenômeno que pode se manifestar em consequência de vários fatores, conforme descritos por MARCELLI (1998).

Os fatores adversos presentes no histórico de vida de crianças com sintomas depressivos estão representados na tabela 2.

TABELA 2. Fatores adversos em escolares, segundo frequência e porcentagem

\begin{tabular}{llr}
\hline Fatores Adversos & F & $\%$ \\
\hline Abuso sexual & 1 & 2,7 \\
\hline Drogas na família & 3 & 8,1 \\
Luto & 3 & 8,1 \\
Mudança de escola & 5 & 13,5 \\
Pais negligentes & 2 & 5,4 \\
Pai ou mãe ausente & 9 & 24,3 \\
Pai preso & 1 & 2,7 \\
Pais rígidos & 1 & 2,7 \\
Relacionamento conturbado na família & 4 & 10,8 \\
Separação dos pais / divorciados & 3 & 8,1 \\
Situações novas & 2 & 5,4 \\
Reprovação de ano & 3 & 8,1 \\
\hline Fonte: Dados de prontuários de uma clínica escola de psicologia, no período de 2011 a 2012.
\end{tabular}

De acordo com a tabela 2, o principal fator adverso identificado nos prontuários foi a ausência do pai ou da mãe em $24,3 \%$ dos casos, seguido por mudança de escola com $13,5 \%$ e relacionamento conturbado na família com $10,8 \%$. O luto, as drogas na família, a separação dos pais e a reprovação de ano apresentaram percentual de $8,1 \%$. Pais negligentes e situações novas apresentaram $5,4 \%$. Abuso sexual, pais rígidos e pai preso obtiveram percentual de $2,7 \%$. 
PINTO et al. (2014) realizaram um estudo que investigou a relação que as dimensões de suporte emocional, rejeição e tentativa de controle estabelecem com o grau de elaboração emocional das crianças. A pesquisa revelou a influência dos pais sobre o processo de ajustamento adaptativo da criança e seu papel na promoção do bem-estar e do desenvolvimento bem-sucedido dos filhos, uma vez que o sentimento de segurança e validação provenientes dos pais proporciona à criança níveis mais elevados de elaboração emocional. Tal fato permite associar porque as crianças deste estudo apresentaram em sua maioria a ausência de pai ou mãe como um fator adverso relacionado aos sintomas depressivos. Uma vez que há falta de um dos genitores, infere-se que também ocorra uma falha nas dimensões de suporte emocional, dificultando a estas crianças a regulação emocional e contribuindo para as manifestações depressivas.

CRUVINEL \& BORUCHOVITCH (2009), em um estudo que objetivou analisar a relação entre sintomas depressivos em crianças e as variáveis familiares, constataram que no grupo de crianças com sintomatologia depressiva os pais apresentaram dificuldades em relação à supervisão da criança. Notaram nesses casos que os pais apresentavam maior fragilidade no monitoramento e supervisão do filho, pois não existia uma rotina quanto às atividades diárias da criança e os integrantes dessas famílias desfrutavam menos tempo juntos. Mediante a literatura exposta, infere-se que pais de crianças depressivas apresentam dificuldades em perceber as necessidades de cuidado e de supervisão que a criança requer. Esses pais, por sua vez, adotam uma postura negligente frente à necessidade do filho, pois não conseguem suprir uma demanda de atenção e monitoramento que a criança necessita nesta fase. Tal fato vem ao encontro do presente estudo, uma vez que a negligência dos pais foi salientada como um fator adverso relacionado ao desenvolvimento dos sintomas depressivos.

CALDERARO \& CARVALHO (2005) estudaram as manifestações depressivas em crianças de três a 10 anos de idade, e verificaram que as famílias analisadas se encontravam em situações de vida muito adversas, permeadas por conflitos conjugais e pela dificuldade dos pais em desempenhar os cuidados afetivos à criança. As consequências da inexistência de cuidados afetivos familiares foram registradas em sete dos nove casos estudados. As pesquisadoras salientam que, uma vez que os pais apresentam dificuldades para conter seus próprios conflitos e angústias internas, dificilmente conseguirão auxiliar seus filhos a ter um desenvolvimento emocional saudável. Tais achados correlacionam-se aos dados deste estudo, pois nos prontuários analisados as famílias apresentavam relacionamento conturbado e separação dos pais.

\section{CONCLUSÃO}

O presente estudo teve como objetivo caracterizar os casos de crianças em idade escolar com sintomas sugestivos de tendência depressiva atendidos por um serviço escola e identificar os fatores adversos relacionados. Com base nos dados coletados, pode-se observar que o principal sintoma depressivo é a falta de interesse em atividades escolares e dificuldade de reter informações e, o maior fator adverso foi a ausência do pai ou da mãe.

Pôde-se constatar que os sintomas depressivos analisados neste estudo evidenciam a complexidade da patologia, já que envolve a manifestação de vários sintomas que vão além do sentimento de tristeza e choro constante, considerados culturalmente como as principais características da depressão. Foi possível confirmar a presença de outros sintomas depressivos, que envolvem desde 
aspectos psicológicos e emocionais, como irritabilidade, embravecimento, isolamento social e timidez, até manifestações comportamentais, como queixas somáticas, perda de apetite e dificuldades do sono.

A presente pesquisa também proporcionou a compreensão dos fatores adversos envolvidos na manifestação da patologia. Evidenciou-se que a maior parte dos fatores adversos estão relacionados ao contexto familiar, pois se constatou a presença de conflitos familiares, a ausência do pai ou da mãe, a separação dos pais e drogas na família. Tal fato sugere a importância que a família exerce no desenvolvimento infantil e como a saúde da criança está associada a seu funcionamento. Em contrapartida, foi possível constatar a presença de fatores externos ao ambiente familiar, como a mudança de escola, reprovação de ano e luto como fatores adversos.

Destaca-se a necessidade de estudos que enfoquem a relação existente entre a dinâmica familiar e a manifestação da depressão infantil. Os achados do presente trabalho sinalizam a necessidade de que a escola e os serviços de saúde estejam preparados para reconhecerem os sintomas da depressão infantil, que tendem a se manifestar no ambiente escolar, buscando implantar ações de promoção da saúde e o tratamento adequado à criança em desenvolvimento. Ressalta-se, também, o papel do suporte familiar como um fator protetivo à depressão infantil.

\section{REFERÊNCIAS}

AMERICAN PSYCHIATRIC ASSOCIATION. Manual diagnóstico e estatístico de transtornos mentais: DSM-5. 5.ed. Porto Alegre: Artmed, 2014.

ANDRIOLA, W. B.; CAVALCANTE, L. R. Avaliação da depressão infantil em alunos da pré- escola. Psicologia Reflexão e Crítica, v. 12, n. 2, p. 419-428, 1999. DOI: 10.1590/S0102-79721999000200011.

ALVARES, A. M.; LOBATO, G. R. Um estudo exploratório da incidência de sintomas depressivos em crianças e adolescentes em acolhimento institucional. Temas em Psicologia, v. 21, n. 1, p. 151-164, 2013. DOI: 10.9788/TP2013.1-11.

BENEVIDES, J.; Sousa, M.; Carvalho, C. B.; Caldeira, S. N. Sintomatologia depressiva e insatisfação escolar. Revista Estudios e Investigación em psicologia y educación, v.Extr, n. 5, 2015. DOI: 10.17979/reipe.2015.0.05.109.

CALDERARO, R. S. S.; CARVALHO, C. V. Depressão na infância: Um estudo exploratório. Psicologia em Estudo, v.10, n.2, p. 181-189, 2005. DOl: 10.1590/S1413-73722005000200004.

CARVALHO, C.; RAMIRES, V. R. R. Repercussões da depressão infantil e materna no brincar de crianças: revisão sistemática. Psicologia: Teoria e Prática, v. 15, n.2, p. 46-61, 2013. Recuperado 20 maio, 2016, de http://editorarevistas.mackenzie.br/index.php/ptp/article/view/4035/4451.

COlAVITE, J.; SILVA, F. F; GARBI, J. P.; SILVA, M. O.; Ribeiro, R. A.; Cardoso, H. F. Depressão: crianças também sofrem com essa doença. Psicologo Informação, v.17, n.17, p.123-131, 2013. DOI: http://dx.doi.org/10.15603/21760969/pi.v17n17p123-131. 
CRUVINEL, M.; BORUCHOVITCH, E. Compreendendo a depressão infantil. Petrópolis, RJ: Vozes, 2014.

Sintomas de depressão infantil e ambiente familiar. Psicologia em pesquisa, v.3, n.1, p. 87-100, 2009. Recuperado 19 maio, 2016, de http://pepsic.bvsalud.org/scielo.php?script=sci_arttext\&pid=S198212472009000100008.

Depressão infantil: Uma contribuição para a prática educacional. Psicologia escolar e educacional, v. 7, n. 1, p. 77-84, 2003. DOI: 10.1590/S141385572003000100008.

FERNANDES, A. M.; MILANI, R. G. A Depressão Infantil, o Rendimento Escolar e a Autoeficácia: Uma Revisão da Literatura. Revista Cesumar - Ciências Humanas e Sociais Aplicadas, v.15, n.2, p.381-403, 2010. Recuperado 18 maio, 2016, de http://periodicos.unicesumar.edu.br/index.php/revcesumar/article/view/932/1116.

FIGUEIREDO, P. C.; MOTTA, I. F. Resgatando o lugar de pais: uma proposta de promoção de saúde mental. Psicologia, Saúde \& Doenças, v.15, n.1, p. 47-59, 2014. Recuperado 18 maio, 2016, de http://www.redalyc.org/articulo.oa?id=36231157006.

LIMA, D. Depressão e doença bipolar na infância e adolescência. Jornal de Pediatria, v.80, n.2, p. S11-S20, 2004. DOI: 10.1590/S0021-75572004000300003.

MARCELLI, D. Manual de psicopatologia da infância Ajuriaguerra. $5^{\circ}$ edição. Porto Alegre: ArtMed, 1998.

MILANI, R. G. O comportamento e o autoconceito de crianças vítimas de violência doméstica. In: Caetano, L. M.; Yaegashi, S. F. R. Relação escola e família: diálogos interdisciplinares para a formação da criança. São Paulo: Paulinas, p. 41-62, 2014.

MIRANDA, M. V.; FIRMO, W. C. A.; CASTRO, N. G.; ALVES, L., P., L.; DIAS, C. N.; RÊGO, M. M.; POPPE, M. C. M.; DIAS, R. S. Depressão infantil: aspectos gerais, diagnóstico e tratamento. Cadernos de Pesquisa, v. 20, n. 3, 2013. Recuperado 17 maio, 2016, de http://www.periodicoseletronicos.ufma.br/index.php/cadernosdepesquisa/article/view/ 2269.

MOMBELLI, M. A.; COSTA, J. B.; MARCON, S. S.; MOURA, C. B. Estrutura e suporte familiar como fatores de risco de stress infantil. Estudos de Psicologia (Campinas), v. 28, n. 3, p. 327-335, 2011. DOI: 10.1590/S141498932001000200007.

PERANDRÉA, M. E. As necessidades emocionais da criança e seu ingresso na educação infantil. In: Bianchini, L. G. et al. (Org.). Psicopedagogia: reflexões sobre família e escola. 1ed.Curitiba: CRV, v. 1, p. 181-190, 2015. 
PINTO, H. M.; CARVALHO, A. R.; SA, E. N. Os estilos educativos parentais e a regulação emocional: Estratégias de regulação e elaboração emocional das crianças em idade escolar. Análise Psicológica, v. 32, n. 4, p. 387-400, 2014. DOI: 1014417/ap.32.3.844.

RAMOS, E. G. Estresse infantil: impactos sobre o desempenho e saúde do escolar. Psicologia em Foco, v. 5, n. 1, 2015. Recuperado 20 maio, 2016, de http://periodicos.piodecimo.edu.br/online/index.php/psicologioemfoco/article/view/148 $/ 187$.

SANTOS, A. J.; MONTEIRO, L.; SOUSA, T.; FERNANDES, C.; TORRES, N.; VAUGHN, B. O reduzido envolvimento social: implicações para o ajustamento psicossocial de crianças em contexto pré-escolar. Psicologia: Reflexão e Crítica, v. 28, n. 1, Mar/2015 p. 186-193. DOI: 10.1590/1678-7153.201528120.

SILVA, M. D.; LACERDA, A. M. Depressão infantil: características e tratamento. Caderno Discente, v. 1, n. 1, 2014. Recuperado 21 maio, 2016, de http://humanae.esuda.com.br/index.php/Discente/article/view/158/84. 